\title{
Second breast-conserving therapy with interstitial brachytherapy (APBI) as a salvage treatment in ipsilateral breast tumor recurrence: a retrospective study of $\mathbf{4 0}$ patients
}

\author{
Salvatore Cozzi, MD!, Dina Najjari Jamal, MD², Andrea Slocker, MD², Maria Laplana, MD², Amparo Garcia Tejedor, MD³, \\ Prof. Marco Krengli, MD4 Prof. Ferran Guedea, MD², Cristina Gutierrez, MD, PhD² \\ 'Department of Radiation Oncology, Humanitas Cancer Center, Rozzano Milano, Italy, 2Department of Radiation Oncology. Catalan Institute \\ of Oncology, University of Barcelona, L'Hospitalet de Llobregat, Barcelona, Spain, ${ }^{3}$ Gynecology and Obstetrics Department, Hospital de \\ Bellvitge, Barcelona, Spain, “Department of Radiation Oncology, "Ospedale Maggiore della Carità", Università degli studi del Pimonte \\ Orientale, Novara, Italy
}

\begin{abstract}
Purpose: Breast-conserving treatment (BCT) have emerged as an alternative to mastectomy in patients with ipsilateral breast tumor recurrence (IBTR). We evaluated survival outcomes and treatment-related toxicity in a series of 40 patients with IBTR, who underwent tumorectomy plus interstitial brachytherapy (APBI) as a salvage treatment.

Material and methods: Retrospective analysis included 40 patients diagnosed with IBTR and treated with intraoperative (26 patients) or post-operative (14 patients) multicatheter brachytherapy for APBI at our institution between June 2002 and October 2017. We assessed cosmesis, toxicity, overall survival (OS), and cancer-specific survival (CSS).

Results: Tumorectomy was performed in all cases, including intraoperative tumor margin assessment and sentinel node biopsy. Median age was 65 years (range, 41-92). The total prescribed dose was 32 Gy (8 fractions) in 19 patients and 34 Gy (10 fractions) in 20 patients. One elderly patient (age 92) received a single fraction of 16 Gy. Median follow-up was 61.5 months (range, 6-153). A median of 14 tubes were inserted. Mean treated $V_{100}$ was 115 cc. Two patients developed a second relapse at 3 and 5 years after salvage treatment: one patient underwent salvage mastectomy and remains alive 10 years after brachytherapy, and the second one developed both local relapse and bone metastasis. The disease is stable at present. Five-year OS and CSS rates were $85.3 \%$ and $97.5 \%$, respectively. Two patients died from cancer-related causes. Acute and late toxicity rates were low; seven patients developed acute infectious mastitis. Late fibrosis > grade 3 and late mastitis were observed in 14 and 6 cases, respectively.

Conclusions: Second BCT with APBI as a salvage treatment in ipsilateral breast recurrence achieves good local disease control, with a satisfactory toxicity profile compared to mastectomy. This approach seems to be safe and effective, although more data from randomized trials are needed.

Key words: APBI, breast cancer, local relapse, brachytherapy, salvage brachytherapy.

\section{Purpose}

Local ipsilateral breast tumor recurrences (IBTR) are relatively uncommon and highly heterogeneous. The risk of IBTR depends on numerous factors, including age, inherited susceptibility, tumor characteristics, type of primary cancer treatment, and lifestyle factors such as obesity and alcohol use. Recurrence rates are approximately $10 \%$ at 10 years and $20 \%$ at 15 years [1]. Several studies $[2,3]$ have sought to identify prognostic factors for recurrent disease after radical treatment for invasive breast cancer. Those studies have found that outcomes are worse in patients who experience a recurrence within two years from the initial treatment compared to those with a longer recurrence-free interval. This difference may, at least partially, be explained by the hypothesis that early recurrences are caused by cell repopulation due to persistent microscopic disease, whereas late recurrences are more likely attributable to be due to a new primary tumor formation.

In recurrent disease, salvage radiotherapy is generally not an option, because most patients who develop locally 
recurrent disease have previously undergone breast irradiation. As a result, until lately, the standard salvage treatment in these cases was mastectomy. However, in recent years, several single-institution studies [4] have investigated the use of breast-conserving surgery (BCS) with or without adjuvant radiotherapy. A literature review conducted by Hannoun-Levi et al. showed that pa-

Table 1. Primary tumor characteristics

\begin{tabular}{|c|c|}
\hline Characteristics & $n(\%)$ \\
\hline \multicolumn{2}{|l|}{ Histological subtype } \\
\hline Ductal invasive & $25(62.5)$ \\
\hline Unknown & $9(22.5)$ \\
\hline Others & $6(15)$ \\
\hline \multicolumn{2}{|l|}{ T stage } \\
\hline pTis & $4(10)$ \\
\hline pT1 & $15(37.5)$ \\
\hline pT2 & $6(15)$ \\
\hline pT3 & $1(2.5)$ \\
\hline Unknown & $14(35)$ \\
\hline \multicolumn{2}{|l|}{ N stage } \\
\hline cNO (sn) & $18(45)$ \\
\hline $\mathrm{pN1}$ & $6(15)$ \\
\hline $\mathrm{pN2}$ & $2(5)$ \\
\hline Unknown & $14(35)$ \\
\hline \multicolumn{2}{|l|}{ Molecular subtype } \\
\hline Luminal A & $10(25)$ \\
\hline Luminal B & $2(5)$ \\
\hline Triple negative & $1(2.5)$ \\
\hline Unknown & $27(67.5)$ \\
\hline \multicolumn{2}{|l|}{ Systemic treatment } \\
\hline Chemotherapy & $10(25)$ \\
\hline Hormonotherapy & $16(40)$ \\
\hline \multicolumn{2}{|c|}{ Previous radiotherapy treatment } \\
\hline $50 \mathrm{~Gy}+\mathrm{BQ}$ boost & $35(87.8)$ \\
\hline $50 \mathrm{~Gy}+$ EBRT boost & $3(7.5)$ \\
\hline Unknown & $2(5)$ \\
\hline \multicolumn{2}{|l|}{ Late toxicity } \\
\hline$\overline{\mathrm{GO}-\mathrm{G} 1}$ & 28 \\
\hline $\mathrm{G} 2$ & 3 \\
\hline$\overline{G 3}$ & 0 \\
\hline Unknown & 9 \\
\hline
\end{tabular}

tients treated with a second breast-conserving treatment $(\mathrm{BCT})$, that is tumorectomy plus radiotherapy, had only a 10\% risk of developing another recurrence at the same site, a rate that is comparable to the recurrence rate observed in patients treated with salvage mastectomy. In both groups, overall survival (OS) was the same (75\% at 10 years) [4]. In addition, a multicentric trial conducted by the GEC-ESTRO Breast Cancer Working Group found that patients who underwent salvage therapy involving lumpectomy plus re-irradiation by interstitial multicatheter brachytherapy, had a recurrence rate for a second IBTR of approximately 5\% [5]. These data suggest that combining BCS with local irradiation may be an emerging standard of care in IBTR, especially when the aim is to preserve the breast. This combined approach is supported by data from several retrospective studies [6], but no randomized trials have yet been performed to determine non-inferiority. In this context, more data to support the safety and efficacy of this approach would be desirable.

The most common approach for re-irradiation in patients undergoing a second BCT is interstitial brachytherapy, either low-dose-rate (LDR) or high-dose-rate (HDR) $[7,8,9,10,11,12]$. However, external beam radiation therapy (EBRT) and intraoperative radiation therapy (IORT) (50 kV photons) have also been described $[13,14]$.

The aim of the present retrospective study was to evaluate the outcomes and treatment-related toxicity in a series of patients who underwent intraoperative or post-operative multicatheter interstitial brachytherapy for locally recurrent breast cancer.

\section{Material and methods}

Between June 2002 and October 2017, 40 patients underwent BCT at our institution (the Catalan Institute of Oncology, Barcelona, Spain) to treat an IBTR. The treatment consisted of a second tumorectomy plus either intraoperative $(n=26)$ or post-operative $(n=14)$ multicatheter brachytherapy implant for accelerated partial breast irradiation (APBI). We retrospectively analyzed overall survival, toxicity, and cosmesis. The main characteristics of the primary tumor are summarized in Table 1 . In all cases, the treatment included tumorectomy, with a resection margin that included sufficient normal breast tissue to ensure clear margins. Sentinel node biopsy (SNB) was performed in all cases, except for patients who had already undergone axillary lymph node dissection for a primary tumor.

Eligibility criteria for multicatheter brachytherapy were as follows: 1) Locally recurrent disease; 2) Breast anatomy suitable for multicatheter implantation; 3) Refusal to undergo mastectomy after previous treatment with BCS and adjuvant whole-breast irradiation (WBI); and 4) Unicentric, unifocal tumor with negative margins and negative SNB. Follow-up consisted of a complete clinical examination and was performed during post-operative stage at first or second month, then every 6 months for the first two years, and annually thereafter. All the patients underwent an annual mammography.

This study was conducted in accordance with our institutional protocols, and all of the cases were presented 
at a breast cancer tumor board. Written informed consent was obtained from every patient prior to treatment.

\section{Implant technique and treatment delivery}

All patients underwent tumorectomy. During the surgical procedure, six metallic clips were inserted around the tumor bed margins following the technique described by Major et al. [15]. Multicatheter implantation was performed intraoperatively in 26 patients and post-operatively in 14 patients. Intraoperative implantation is presented in Figure 1. After tumorectomy, tissue samples from the sentinel lymph node and tumor margins were sent for intraoperative pathological evaluation. After the pathological report confirmed a negative result, we proceeded to manually insert metallic needles into the open cavity following the technique described by our group previously [16]. Post-operative implantation is presented in Figure 2.

In the 14 patients who underwent post-operative brachytherapy, this procedure was performed from 15-45 days after tumorectomy under direct visualization of the seroma using ultrasound guidance. In all cases, a plastic guide template with needle holes was used to achieve geometric distribution. The needles were spaced to form equilateral triangles of $1.6 \mathrm{~cm}$ and inserted in two to four planes. After insertion, the needles were replaced with plastic tubes. The number of applicators and tubes varied on a case by case basis according to the size of the tumor cavity and individual breast anatomy. Next, a computed tomography (CT) scan with $2 \mathrm{~mm}$ slice thickness was performed. The Oncentra system (Elekta Company; Veenendaal, the Netherlands) was used to define the planning target volume (PTV), including a $2 \mathrm{~cm}$ margin of safety to the tumor edge [15]. In cases in which the PTV overlapped the skin and/or chest wall, a $5 \mathrm{~mm}$ margin was cropped out of these structures [17]. A modified Paris system was used for dosimetric purposes [18]. The treatment planning parameters were as follows: $\geq 90 \%$ of the defined PTV had to receive $100 \%$ of the prescribed dose (coverage ratio $\geq 0.9$ ); maximum dose to the skin:
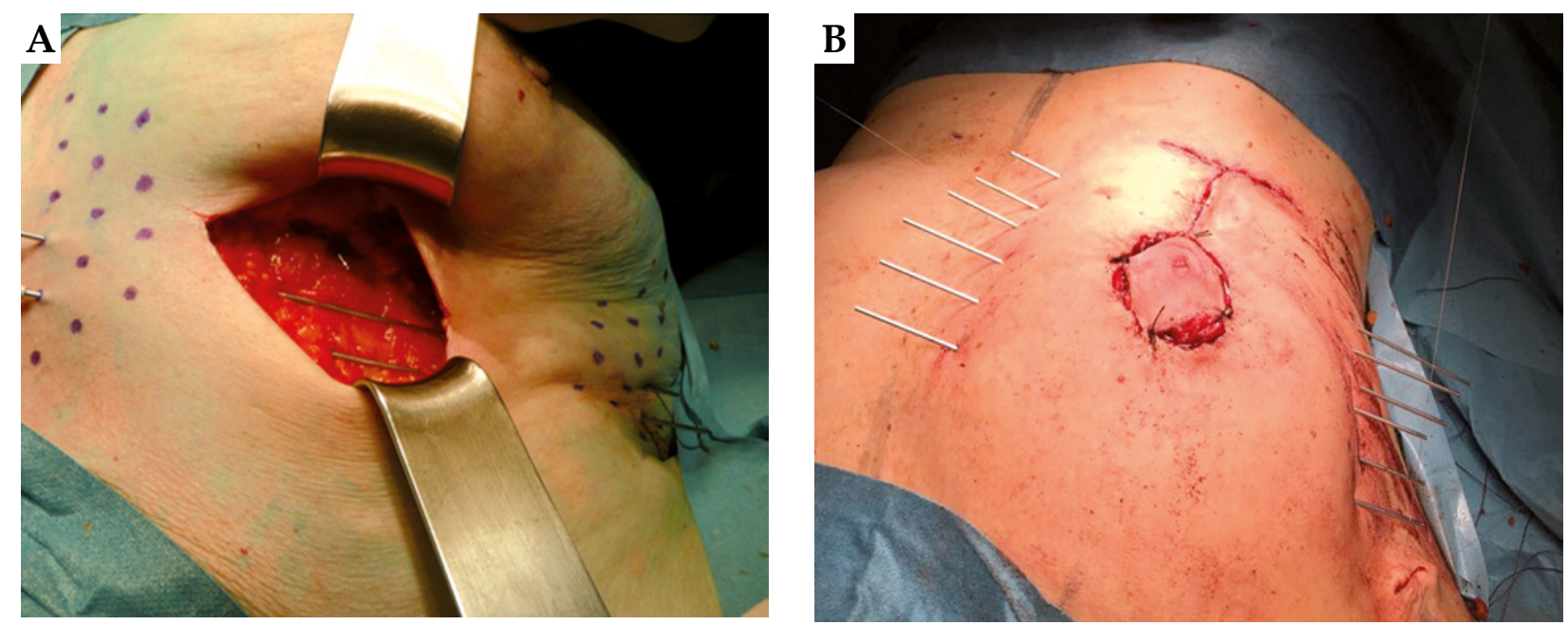

Fig. 1. Intraoperative implantation: A) Manual insertion of metallic needles into the open cavity; B) Needles inserted in the inferior plane and sutured skin
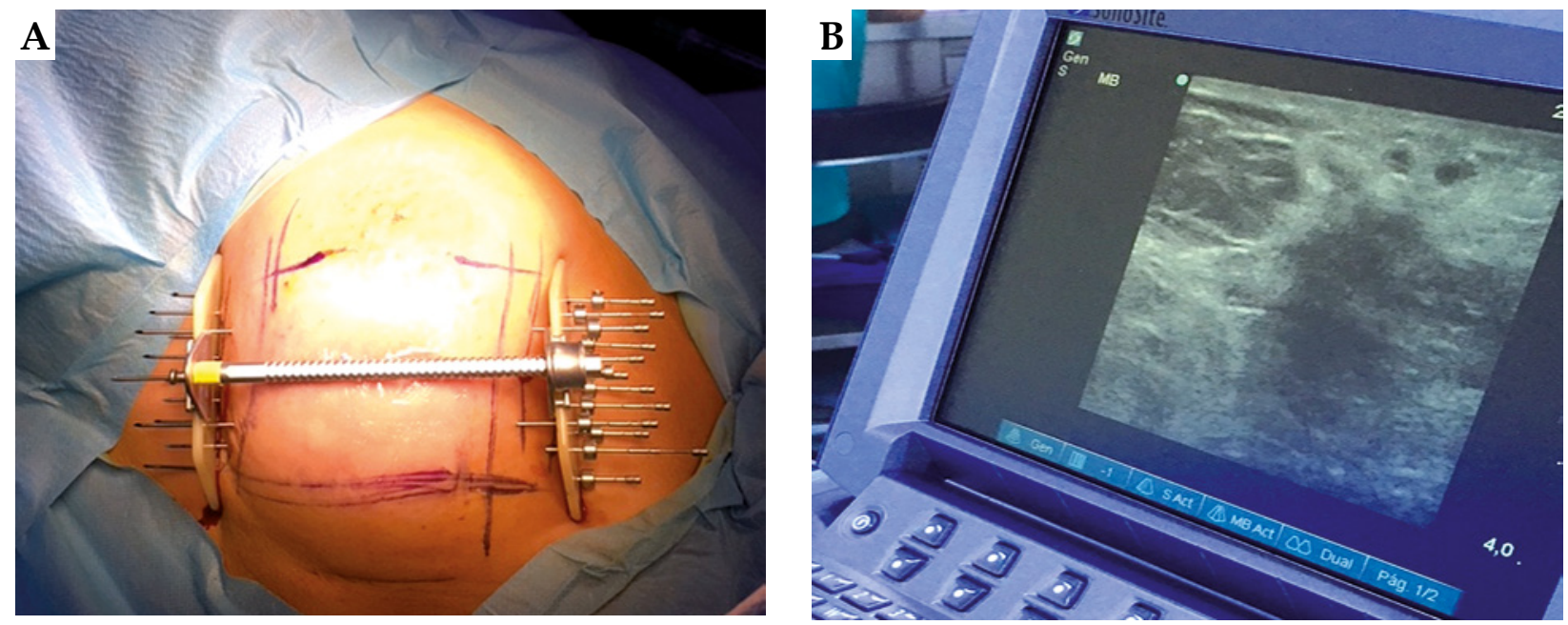

Fig. 2. Post-operative implantation: A) The needles were spaced to form equilateral triangles of $1.6 \mathrm{~cm}$ using a plastic template; B) Direct visualization of the seroma using ultrasound guidance 
$\leq 70 \%$ of the prescribed dose; $\mathrm{D}_{90}>100 \% ; \mathrm{V}_{150}<50 \%$, with a dose non-uniformity ratio (DNR) $<0.35$.

Until September 2012, we used the treatment scheme (3.4 Gy/10 fractions) described by Vicini et al. [19]. However, this treatment scheme was changed in October 2012, as our center participated in the GEC-ESTRO randomized trial [20]. Accordingly, the patients in this study received either 10 fractions of 3.4 Gy $(n=20)$ or 8 fractions of 4 Gy $(n=19)$. One elderly patient (age 92$)$ received a single $16 \mathrm{~Gy}$ fraction due to her advanced age and poor performance status [21,22]. Notwithstanding these differences in fractionation schedules, the equivalent total dose in

Table 2. Patient demographics and characteristics of recurrent tumor

\begin{tabular}{|c|c|}
\hline Parameter & $n(\%)$ \\
\hline \multicolumn{2}{|l|}{ Follow-up (months) } \\
\hline Median & 61.5 \\
\hline Range & $6-153$ \\
\hline \multicolumn{2}{|c|}{ Time between primary cancer and recurrence (years) } \\
\hline Median & 10.5 \\
\hline Range & $2-29$ \\
\hline \multicolumn{2}{|l|}{ Age (years) } \\
\hline Median & 65 \\
\hline Range & $41-92$ \\
\hline $41-59$ & $14(35)$ \\
\hline $60-69$ & $11(27.5)$ \\
\hline$>70$ & $15(37.5)$ \\
\hline \multicolumn{2}{|l|}{ Histological subtype } \\
\hline Ductal invasive & $26(65)$ \\
\hline Carcinoma in situ & $8(20)$ \\
\hline Mucinous invasive & $2(5)$ \\
\hline Papillary invasive & $3(7.5)$ \\
\hline Lobular invasive & $1(2.5)$ \\
\hline \multicolumn{2}{|l|}{ Intrinsic subtype } \\
\hline Luminal A & $28(70)$ \\
\hline Luminal B & $8(20)$ \\
\hline ErB2 overexpression & $3(7.5)$ \\
\hline "Basal Like" & $1(2.5)$ \\
\hline \multicolumn{2}{|l|}{ Grading } \\
\hline 1 & $9(22.5)$ \\
\hline 2 & $13(32.5)$ \\
\hline 3 & $11(27.5)$ \\
\hline Unknown & $7(17.5)$ \\
\hline
\end{tabular}

2 Gy fractions $\left(\mathrm{EQD}_{2}\right)$, with a tumor $\alpha / \beta$ ratio value of 4 was similar in both schemes (41.93 Gy and $42.67 \mathrm{~Gy}$, respectively), as were the biologically-effective doses (BEDs): $62.9 \mathrm{~Gy}$ and $64 \mathrm{~Gy}$, respectively. The $\mathrm{EQD}_{2}$ for a single fraction of $16 \mathrm{~Gy}$ is equal to $53 \mathrm{~Gy}[23,24]$.

\section{Results}

A total of 40 women diagnosed with IBTR underwent salvage HDR brachytherapy after BCS. Of these 40 patients, 26 (65\%) underwent intraoperative interstitial multicatheter implant, while the other 14 patients (35\%) received a post-operative implant. Median follow-up was 61.5 months (range, 12-153). Median age at recurrence was 65 years (range, 41-92). Most patients (25/40, 62.5\%) were $\geq 60$ years. The demographic and tumor characteristics are provided in Table 2. The median time to local recurrence (LR) was 10 years (range, 2-29). In one patient, the recurrence-free interval was 29 years; in that case, the recurrence was located at the edge of the primary surgical bed and was interpreted as a late relapse rather than as a new primary cancer in the same quadrant. Most patients $(n=26,65 \%)$ had infiltrating ductal carcinoma (Table 2), and $70 \%$ had luminal A disease. A median of 14 tubes (range, 7-18) were inserted. In almost all cases $(70 \%), 3$ catheter planes were used. Nineteen patients (47.5\%) were treated according to the revised GEC-ESTRO treatment schedule (32 Gy in 8 fractions), 20 patients $(50 \%)$ were treated with the classic schedule (34 Gy in 10 fractions), and one patient received $16 \mathrm{~Gy}$ in a single faction. The mean $\mathrm{V}_{100}$ was $115 \mathrm{cc}$, with a median of $132.5 \mathrm{cc}$ (range, $54.8-164 \mathrm{cc}$ ). Mean $\mathrm{V}_{150}$ was $38.5 \mathrm{cc}$ (range, 16-65 cc). Treatment characteristics and parameters of the dosimetric analysis are provided in Table 3. At least $90 \%$ of the defined PTV received $100 \%$ of the prescribed dose (coverage index $>0.9$ ). Underdosing of the PTV was accepted only to meet dose constraints for the skin and chest wall. Mean coverage index was $90.1 \%$ (median, $92.25 \%, \min 78.61, \max 99 \%)$.

\section{Disease control}

In our series, two patients developed a second relapse at 3 and 5 years after salvage treatment, respectively. One patient underwent salvage mastectomy and remains free of disease and alive 10 years after brachytherapy. The second one developed both local relapse and bone metastasis. She was treated with systemic therapy; the disease is stable at present. Overall survival at 3 and 5 years were $97 \%$ and $85.3 \%$, respectively. Five-year CSS was $96 \%$. The rate of local relapse (local relapse-free survival), evaluated with Kaplan-Meier analysis was $96.6 \%$ at 5 years and $91.7 \%$ at 7 years. Three patients developed distant metastasis: one at 1 month, second at 3 years, and third patient at 10 years. Five-year metastasis-free survival at 3 and 5 years was $94 \%( \pm 8 \%)$. There were two cancerrelated deaths, which was due to metastatic disease 4 and 10 years after salvage treatment. Bone and lymph node metastases were detected in one patient 30 days after salvage treatment, a finding that suggests the patient had been understated at the initial surgery. At the time of 
writing the paper, this patient remains alive, currently undergoing chemotherapy. A total of 3 patients $(7.5 \%)$ died of causes unrelated to cancer. No evidence of relapse was observed in most cases (34/40 patients, $85 \%)$.

\section{Toxicity and cosmetic outcomes}

One patient developed a G2 hematoma. Acute infectious mastitis was observed in 7 patients (17.5\%). Due to this relatively high infection rate, prophylactic antibiotics are now routinely administered at our institution for all intraoperative approaches. None of the patients who underwent post-operative catheter implantation developed an infection, even without the use of prophylactic antibiotics. Grade $(G) 0-2$ late fibrosis was observed in $65 \%$ of the patients, while $27.5 \%(n=11)$ and $7.5 \%$ $(n=3)$ developed G3 and G4 late fibrosis, respectively. At the last follow-up visit, late toxicity in the series was as follows: mastitis (6 cases, 15\%), hypochromic skin spots at the catheter entrance and exit points (8 patients, $20 \%$ ), telangiectasia (7 patients, $17.5 \%$ ), and fat necrosis (one case). Cosmetic outcomes determined according to on the 4-point Harvard breast cosmesis scale [25] were as follows: good $(n=23,57.5 \%)$, fair $(n=6,15 \%)$, and poor $(n=7,17.5 \%)$; in 4 cases, no data were available $(10 \%)$. Acute and late toxicity outcomes are shown in Table 4. Unfortunately, we do not have any data on cosmetic outcomes after primary treatment.

\section{Discussion}

The present retrospective study describes survival and treatment-related toxicity outcomes in a series of 40 patients who underwent tumorectomy followed by either intraoperative or post-operative multicatheter brachytherapy as a salvage treatment for IBTR. Our findings show that survival outcomes using this technique are good, with OS and CSS rates at 5 years of $85.3 \%$ and $96 \%$, respectively. Importantly, only two patients in this series died of cancer-related causes, and only one developed local relapse and bone metastasis. In the last 30 years, several studies have been performed to validate the use of a second BCT in patients with IBTR. While mastectomy is still widely used for salvage treatment, there is a growing trend towards breast-conserving approaches consisting of tumorectomy plus re-irradiation (particularly APBI). The available data suggest that this approach is a valid alternative to mastectomy $[26,27,28]$. Although no non-inferiority phase III studies are currently available, there is a growing body of evidence indicating that re-treatment with breast conserving procedures is both safe and effective, with excellent local control, survival, cosmesis, and patient's satisfaction $[26,27,28]$.

One of the main concerns in patients with IBTR is the risk of developing metastatic disease. The degree of risk appears to be associated with tumor-related prognostic factors and with the time elapsed between diagnosis of the original breast cancer and disease recurrence, which is considered an independent prognostic factor. Previous hormonotherapy or chemotherapy are also associated with the risk of developing systemic disease $[3,29]$. Inter- estingly, the overall risk of developing distant metastases is approximately $30 \%$, even in patients who undergo mastectomy or mastectomy plus re-irradiation. Overall survival rates range from $31 \%$ to $80 \%[2,30,31,32,33]$.

Table 3. Treatment characteristics and dosimetric analysis

Parameter

\begin{tabular}{lc}
\hline Doses and fractionation & \\
\hline $34 \mathrm{~Gy} / 10 \mathrm{Fr}$ & $n=20(50 \%)$ \\
\hline $32 \mathrm{~Gy} / 8 \mathrm{Fr}$ & $n=19(47.5 \%)$ \\
\hline $16 \mathrm{~Gy}$ in single fraction & $n=1(2.5 \%)$ \\
\hline Number of catheter plans & 3 \\
\hline Median & $1-4$
\end{tabular}

Number of catheters

\begin{tabular}{ll}
\hline Median 14 \\
\hline
\end{tabular}

Range 7-18

Mean volume $\left(\mathrm{cm}^{3}\right)$

\begin{tabular}{lc}
\hline$V_{100}$ & 115 \\
\hline$V_{150}$ & 38.5
\end{tabular}

Median volume $\left(\mathrm{cm}^{3}\right)$

\begin{tabular}{lr}
\hline $\mathrm{V}_{100}$ & 132.5 \\
\hline $\mathrm{V}_{150}$ & 37
\end{tabular}

Minimum volume $\left(\mathrm{cm}^{3}\right)$

\begin{tabular}{lc}
\hline$V_{100}$ & 54.8 \\
\hline$V_{150}$ & 16.4
\end{tabular}

Maximum volume $\left(\mathrm{cm}^{3}\right)$

\begin{tabular}{lc}
\hline $\mathrm{V}_{100}$ & 164 \\
\hline $\mathrm{V}_{150}$ & 65.2 \\
\hline PTV volume $\left(\mathrm{cm}^{3}\right)$ & 115 \\
\hline Mean & $93-137$ \\
\hline Range & \\
\hline Coverage index (CI) (\%) & 90.1 \\
\hline Mean & 92.25 \\
\hline Median & $78.61-99$ \\
\hline
\end{tabular}

Dose non-uniformity ratio (DNR)

\begin{tabular}{lc}
\hline Mean & 0.28 \\
\hline Median & 0.27 \\
\hline Range & $0.16-0.40$
\end{tabular}


Table 4. Acute and late toxicity profile

$n(\%)$

\begin{tabular}{lc}
\hline Acute toxicity & \\
\hline Infectious mastitis & $7(17.5)$ \\
\hline Hematoma & $1(2.5)$ \\
\hline Late toxicity & $26(65)$ \\
\hline Fibrosis G0-G2 & $11(27.5)$ \\
\hline Fibrosis G3 & $3(7.5)$ \\
\hline Fibrosis G4 & $6(15)$ \\
\hline Mastitis & $8(20)$ \\
\hline Hypochromic skin spots & $5(12.5)$ \\
\hline Telangiectasia & $7(17.5)$ \\
\hline Fat necrosis & $1(2.5)$
\end{tabular}

In our series, three patients developed a second relapse after salvage treatment. Published reports indicate that anywhere from $0 \%$ to $26 \%$ of patients will develop a second local recurrence after BCS with re-irradiation, with a 5-year disease-free survival (DFS) rate of approximately $60 \%$ (range, $31 \%$ to $85 \%$ ). Voogd et al. [30] retrospectively evaluated 266 patients with IBRT treated by mastectomy alone and finding that $25 \%$ of patients developed a second local recurrence. In our series, the 5 -year local control was higher than $95 \%$. The results reported in the study conducted by the GEC-ESTRO Breast Cancer Working Group suggest that BCT with interstitial brachytherapy is feasible and effective in preventing second local recurrences, and that this approach achieves OS, DFS, and local recurrence rates that are at least equivalent to those obtained with salvage mastectomy.

In our series, we inserted a median of 14 catheters, with an average PTV volume of 115 cc. We used large PTV volumes to ensure better coverage of the tumor bed, in accordance with the report by Hannoun-Levi et al. [8] who found that the second local recurrence rate was lower in patients who received higher delivered doses with a larger irradiated volume.

\section{Cosmesis}

Cosmetic outcomes and fibrosis were slightly worse in our series compared to other reports. However, these bad cosmesis results can be explained by the fact that our patients were treated for recurrent disease, which means they had already undergone two surgeries plus adjuvant external WBI. Although cosmesis was considered satisfactory in most of the patients, our results suggest that patients with locally-recurrent disease should be informed that cosmetic outcomes may be less than optimal with this technique. Nevertheless, patients - and clinicians - may consider this to be an acceptable trade-off given the good survival rates achieved with this treatment approach, es- pecially since the only alternative is mastectomy (with or without reconstructive surgery). Although several cases of acute and chronic mastitis were observed in our series, these were successfully managed with supportive therapy without need for additional surgery.

\section{Study strengths and limitations}

The main limitations of this study are the retrospective study design and the relatively small sample size. Another limitation is the lack of a homogenous treatment scheme, which was modified during the study period. Nonetheless, the $\mathrm{EQD}_{2}$ was equivalent.

\section{Conclusions}

The use of the APBI for primary breast cancer is well-established in clinical practice. In patients who develop ipsilateral breast cancer recurrence, the available evidence shows that a second conservative treatment should be considered in well-selected patients. The data reported in this study support the feasibility and safety of APBI, with multicatheter interstitial brachytherapy as a salvage treatment. The body of evidence from this and other studies suggests that this breast-conserving treatment approach is a reasonable alternative to mastectomy. In our series, local control, survival, and toxicity outcomes were good. Although cosmetic outcomes were less than fully satisfactory in some patients, the only alternative that these patients have is mastectomy. Salvage brachytherapy after tumorectomy seems to be safe and effective. However, randomized trials are needed to compare this approach to mastectomy.

\section{Acknowledgements}

The authors with to thank Bradley Londres for editing the manuscript and Dr. Joan Pera for his help with the statistics.

\section{Disclosure}

Authors report no conflict of interest.

\section{References}

1. Brewster AM, Hortobagyi GN, Broglio KR et al. Residual risk of breast cancer recurrence 5 years after adjuvant therapy. J Natl Cancer Inst 2008; 100: 1179-1183.

2. Salvadori B, Marubini E, Miceli R et al. Reoperation for locally recurrent breast cancer in patients previously treated with conservative surgery. Br J Surg 1999; 86: 84-87.

3. Lee JH, Lee SK, Park SM et al. Independent prognostic factor for overall survival after salvage operation for ipsilateral breast tumor recurrence following breast-conserving surgery. J Breast Cancer 2015; 18: 386-393.

4. Hannoun-Levi JM, Ihrai T, Courdi A. Local treatment options for ipsilateral breast tumour recurrence. Cancer Treat Rev 2013; 39: 737-741.

5. Hannoun-Levi JM, Resch A, Gal J et al. Accelerated partial breast irradiation with interstitial brachytherapy as second conservative treatment for ipsilateral breast tumour recurrence: multicentric study of the GEC-ESTRO Breast Cancer Working Group. Radiother Oncol 2013; 108: 226-231. 
6. Trombetta M, Hannoun-Levi JM. Treatment of second ipsilateral breast tumor event: A need for a new type of evidence for avoiding mastectomy. Eur J Surg Oncol 2017; 43: 849-850.

7. Maulard C, Housset M, Brunel P et al. Use of perioperative or split-course interstitial brachytherapy techniques for salvage irradiation of isolated local recurrences after conservative management of breast cancer. Am J Clin Oncol 1995; 18: 348-352.

8. Hannoun-Levi JM, Houvenaeghel G, Ellis S et al. Partial breast irradiation as second conservative treatment for local breast cancer recurrence. Int J Radiat Oncol Biol Phys 2004; 60: 1385-1392.

9. Chadha M, Feldman S, Boolbol S et al. The feasibility of a second lumpectomy and breast brachytherapy for localized cancer in a breast previously treated with lumpectomy and radiation therapy for breast cancer. Brachytherapy 2008; 7: 22-28.

10. Trombetta M, Julian TB, Bhandari T et al. Breast conservation surgery and interstitial brachytherapy in the management of locally recurrent carcinoma of the breast: the Allegheny General Hospital experience. Brachytherapy 2008; 7: 29-36.

11. Guix B, Lejárcegui JA, Tello JI et al. Exeresis and brachytherapy as salvage treatment for local recurrence after conservative treatment for breast cancer: results of a ten-year pilot study. Int J Radiat Oncol Biol Phys 2010; 78: 804-810.

12. Hannoun-Levi JM, Castelli J, Plesu A et al. Second conservative treatment for ipsilateral breast cancer recurrence using high-dose rate interstitial brachytherapy: preliminary clinical results and evaluation of patient satisfaction. Brachytherapy 2011; 10: 171-177.

13. Deutsch M. Repeat high-dose external beam irradiation for inbreast tumor recurrence after previous lumpectomy and whole breast irradiation. Int J Radiat Oncol Biol Phys 2002; 53: 687-691.

14. Kraus-Tiefenbacher U, Bauer L, Scheda A et al. Intraoperative radiotherapy (IORT) is an option for patients with localized breast recurrences after previous external-beam radiotherapy. BMC Cancer 2007; 7: 178.

15. Major T, Gutiérrez C, Guix B et al. Recommendations from GEC ESTRO Breast Cancer Working Group (II): Target definition and target delineation for accelerated or boost partial breast irradiation using multicatheter interstitial brachytherapy after breast conserving open cavity surgery. Radiother Oncol 2016; 118: 199-204.

16. Cozzi S, Laplana M, Najjari D et al. Advantages of intraoperative implant for interstitial brachytherapy for accelerated partial breast irradiation either frail patients with early-stage disease or in locally recurrent breast cancer. J Contemp Brachytherapy 2018; 10: 97-104.

17. Strnad V, Hannoun-Levi JM, Guinot JL et al. Recommendations from GEC ESTRO Breast Cancer Working Group (I): Target definition and target delineation for accelerated or boost partial breast irradiation using multicatheter interstitial brachytherapy after breast conserving closed cavity surgery. Radiother Oncol 2015; 115: 342-348.

18. Strnad V, Potter R, Kovacs G. Practical Handbook of Brachytherapy. UNI-MED, Verlag AG, Bremen 2014.

19. Vicini F, Beitsch PD, Quiet CA et al. Three-year analysis of treatment efficacy, cosmesis, and toxicity by the American Society of Breast Surgeons MammoSite Breast Brachytherapy Registry Trial in patients treated with accelerated partial breast irradiation (APBI). Cancer 2008; 112: 758-766.

20. Strnad V, Ott OJ, Hildebrandt G et al. 5-year results of accelerated partial breast irradiation using sole interstitial multicatheter brachytherapy versus whole-breast irradiation with boost after breast conserving surgery for low-risk invasive and in-situ carcinoma of the female breast: a randomised, phase 3, non-inferiority trial. Lancet 2016; 387: 229-238.

21. Hannoun-Lévi JM, Cham Kee DL, Gal J et al. Accelerated partial breast irradiation for suitable elderly women using a single fraction of multicatheter interstitial high-dose-rate brachytherapy: Early results of the Single-Fraction Elderly Breast Irradiation (SiFEBI) Phase I/II trial. Brachytherapy 2017; 17: 407-414.

22. Latorre JA, Galdós P, Buznego LA et al. Accelerated partial breast irradiation in a single $18 \mathrm{~Gy}$ fraction with highdose-rate brachytherapy: preliminary results. J Contemp Brachytherapy 2018; 10: 58-63.

23. Dutreix J, Cosset JM, Girinsky T. Biological equivalency of high single doses used in intraoperative irradiation. Bull Cancer Radiother 1990; 77: 125-134.

24. Wheldon TE, Deehan C, Wheldon EG, Barrett A. The linear-quadratic transformation of dose-volume histograms in fractionated radiotherapy. Radiother Oncol 1998; 46: 285-295.

25. Vrieling C, Collette L, Bartelink E et al. Validation of the methods of cosmetic assessment after breast-conserving therapy in the EORTC "boost versus no boost" trial. Int J Radiat Oncol Biol Phys 1999; 45: 667-676.

26. Hannoun-Levi JM, Resch A, Gal J et al.; GEC-ESTRO Breast Cancer Working Group. Accelerated partial breast irradiation with interstitial brachytherapy as second conservative treatment for ipsilateral breast tumour recurrence: multicentric study of the GEC-ESTRO Breast Cancer Working Group. Radiother Oncol 2013; 108: 226-231.

27. Chadha M, Trombetta M, Boolbol S et al. Managing a small recurrence in the previously irradiated breast: is there a second chance for breast conservation? Oncology (Williston Park) 2009; 23: 933-940.

28. Trombetta M, Julian TB, Kim Y et al. Mature follow-up of low dose rate brachytherapy following ipsilateral breast tumor recurrence in patients initially treated with breast conservation therapy. J Solid Tumors 2012; 2: 8-15.

29. Borner M, Bacchi M, Goldhirsch A et al. First isolated locoregional recurrence following mastectomy for breast cancer: results of a phase III multicenter study comparing systemic treatment with observation after excision and radiation. Swiss Group for Clinical Cancer Research. J Clin Oncol 1994; 12: 2071-2077.

30. Voogd AC, van Tienhoven G, Peterse HL et al. For the Dutch Study Group on Local Recurrence after Breast Conservation. Local recurrence after breast conservation therapy for early stage breast carcinoma: detection, treatment, and outcome in 266 patients. Cancer 1999; 85: 437-446.

31. Doyle T, Schultz DJ, Peters C et al. Long-term results of local recurrences after breast conservation treatment for invasive breast cancer. Int J Radiat Oncol Biol Phys 2001; 51: 74-80.

32. Huang E, Buchholz TA, Meric F et al. Classifying local disease recurrences after breast conservation therapy based on location and histology: new primary tumors have more favorable outcomes than true local disease recurrences. Cancer 2002; 95: 2059-2067.

33. Alpert TE, Kuerer HM, Arthur DW et al. Ipsilateral breast tumor recurrence after breast conservation therapy: outcomes of salvage mastectomy vs. salvage breast-conserving surgery and prognostic factors for salvage breast preservation. Int J Radiat Oncol Biol Phys 2005; 63: 845-851. 